\title{
Multi-valued solutions to Hessian equations
}

\author{
Limei Dai and Jiguang Bao
}

\begin{abstract}
In this paper, we use the Perron method to prove the existence of bounded multi-valued viscosity solutions to Hessian equations and interior Lipschitz continuity of the multi-valued solutions.
\end{abstract}

Mathematics Subject Classification (2000). 35J60.

Keywords. Hessian equations, Multi-valued solutions, Viscosity solutions, Existence.

\section{Introduction}

In this paper, we study the multi-valued solutions of the Hessian equation

$$
\sigma_{l}\left(\lambda\left(D^{2} u\right)\right)=f(x),
$$

where $\sigma_{l}(\lambda)$ denotes the $l$ th elementary symmetric function of $\lambda=\left(\lambda_{1}, \ldots, \lambda_{n}\right)$, which is defined by

$$
\sigma_{l}(\lambda)=\sum_{i_{1}<\cdots<i_{l}} \lambda_{i_{1}} \cdots \lambda_{i_{l}}, \quad l=1, \ldots, n,
$$

$\lambda=\lambda\left(D^{2} u\right)$ is the eigenvalues of the Hessian matrix $D^{2} u$ and $f(x)$ is a given nonnegative function. For $l=1(1)$ is Poisson equation $\Delta u=f(x)$, and for $l=n(1)$ is Monge-Ampère equation $\operatorname{det}\left(D^{2} u\right)=f(x)$.

From the theory of analytic functions, we know the typical twodimensional examples of multi-valued harmonic functions are

$$
\begin{aligned}
& u_{1}(z)=\operatorname{Re}\left(z^{\frac{1}{k}}\right), \quad z \in \mathbb{C} \backslash\{0\}, \\
& u_{2}(z)=\operatorname{Arg}(z), \quad z \in \mathbb{C} \backslash\{0\},
\end{aligned}
$$

and

$$
u_{3}(z)=\operatorname{Re}(\sqrt{(z-1)(z+1)}), \quad z \in \mathbb{C} \backslash\{ \pm 1\} .
$$

By 1970s, Almgren [1] had realized that a minimal variety near a multiplicity-k disk could be well approximated by the graph of a multi-valued function minimizing a suitable analog of the ordinary Dirichlet integral. Many facts about harmonic functions are also true for these Dirichlet minimizing 
multi-valued functions. Evans [6-8], Levi [16] and Caffarelli [2,3] studied the multi-valued harmonic functions. Evans [7] proved that the conductor potential of a surface with minimal capacity was a double-valued harmonic function. In [3], Caffarelli proved the Hölder continuity of the multi-valued harmonic functions.

At the beginning of this century, the multi-valued solutions of the Eikonal equation were considered in $[10,11]$, respectively. Later, Jin, etc provided a level set method for the computation of multi-valued geometric solutions to general quasilinear PDEs and multi-valued physical observables to the semiclassical limit of the Schrödinger equations (see [14,15]).

Recently, Caffarelli and Li have investigated the multi-valued solutions of Monge-Ampère equation in [4] where they first introduced the geometric situation of the multi-valued solutions and then obtained the existence, boundedness, regularity and the asymptotic behavior at infinity of the multi-valued viscosity solutions. The multi-valued solutions for the Dirichlet problem of Monge-Ampère equation on exterior planar domains are discussed by Ferrer et al. [9] using complex variable methods.

The geometric situation of the multi-valued functions is given in [4]. Let $n \geq 2, D \subset \mathbb{R}^{n}$ be a bounded domain with smooth boundary $\partial D$, and let $\Sigma \subset D$ be homeomorphic in $\mathbb{R}^{n}$ to an $n$-1-dimensional closed disc, i.e. there exists a homeomorphism $\psi: \mathbb{R}^{n} \rightarrow \mathbb{R}^{n}$ such that $\psi(\Sigma)$ is an $n$-1-dimensional closed disc. Let $\Gamma=\partial \Sigma$, the boundary of $\Sigma$. Thus $\Gamma$ is homeomorphic to an $n$-2-dimensional sphere for $n \geq 3$.

Let $\mathbb{Z}$ be the set of integer and

$$
M=(D \backslash \Gamma) \times \mathbb{Z}
$$

denote a covering of $D \backslash \Gamma$ with the following standard parameterization: fixing an $x^{*} \in D \backslash \Gamma$, and connecting $x^{*}$ by a smooth curve in $D \backslash \Gamma$ to a point $x$ in $D \backslash \Gamma$. If the curve goes through $\Sigma m \geq 0$ times in the positive direction (fixing such a direction), then we arrive at $(x, m)$ in $M$. If the curve goes through $\Sigma m \geq 0$ times in the negative direction, then we arrive at $(x,-m)$ in $M$.

For $k=2,3, \ldots$, we introduce an equivalence relation " $k$ " on $M$ as follows: $(x, m)$ and $(y, j)$ in $M$ are " $\sim k$ " equivalent if $x=y$ and $m-j$ is an integer multiple of $k$. We let

$$
M_{k}:=M / \sim k
$$

denote the $k$-sheet cover of $D \backslash \Gamma$, and let

$$
\partial^{\prime} M_{k}:=\bigcup_{m=1}^{k}(\partial D \times\{m\}) .
$$

For $n=2$, we can understand the covering space $M_{k}$ more clearly from the above example $u_{3}$. In this example, $\Gamma=\{1,-1\}$ and $\Sigma$ is the interval $(-1,1)$. Each time the point $z$ goes around -1 or 1 , it crosses the interval $(-1,1)$ one time.

We define a distance in $M_{k}$ as follows: for any $(x, m),(y, j) \in M_{k}$, let $l((x, m),(y, j))$ denote a smooth curve in $M_{k}$ which connects $(x, m)$ and $(y, j)$, 
and let $|l((x, m),(y, j))|$ denote its length. Define

$$
d((x, m),(y, j))=\inf _{l}|l((x, m),(y, j))|,
$$

where the inf is taken over all smooth curves connecting $(x, m)$ and $(y, j)$. Then $d((x, m),(y, j))$ is a distance.

Definition 1.1. We call a function $u$ is continuous at $(x, m)$ in $M_{k}$ if

$$
\lim _{d((x, m),(y, j)) \rightarrow 0} u(y, j)=u(x, m),
$$

and $u \in C^{0}\left(M_{k}\right)$ if for any $(x, m) \in M_{k}, u$ is continuous at $(x, m)$.

Similarly we can define $u \in C^{\alpha}\left(M_{k}\right), C^{0,1}\left(M_{k}\right)$ and $C^{2}\left(M_{k}\right)$.

To our best knowledge, there are not any results of the multi-valued solutions to Hessian equations. In this paper, we study the multi-valued solutions of the Hessian equation with Dirichlet boundary condition:

$$
\begin{aligned}
\sigma_{l}\left(\lambda\left(D^{2} u\right)\right) & =f(x, m), \quad(x, m) \in M_{k}, \\
u & =\varphi_{m}(x), \quad(x, m) \in \partial^{\prime} M_{k},
\end{aligned}
$$

where $f$ and $\varphi_{1}, \ldots, \varphi_{k}$ satisfy the conditions:

$\left(H_{1}\right) f \in C^{0}\left(M_{k}\right)$, and $0 \leq f \leq b$ for some positive constant $b$.

$\left(H_{2}\right) \varphi_{1}, \ldots, \varphi_{k} \in C^{0}(\partial D)$.

We shall extend some results of the Laplace equation and the MongeAmpère equation to the Hessian equation.

To work in the realm of elliptic equations, we have to restrict the class of functions and domains. Let

$$
\Gamma_{l}=\left\{\lambda \in \mathbb{R}^{n} \mid \sigma_{j}(\lambda)>0, j=1,2, \ldots, l\right\} .
$$

$\Gamma_{l}$ is symmetric, that is, any permutation of $\lambda$ is in $\Gamma_{l}$ if $\lambda \in \Gamma_{l}$. When $l=1$, $\Gamma_{l}$ is the half space $\left\{\lambda \in \mathbb{R}^{n} \mid \lambda_{1}+\lambda_{2}+\cdots+\lambda_{n}>0\right\}$. When $l=n, \Gamma_{l}$ is the positive cone $\Gamma^{+}=\left\{\lambda \in \mathbb{R}^{n} \mid \lambda_{i}>0, i=1, \ldots, n\right\}$. Following [5], we give two definitions.

Definition 1.2. A function $u \in C^{2}\left(M_{k}\right)$ is called $l$-convex if $\lambda(x, m) \in \overline{\Gamma_{l}}$ in $M_{k}$, where $\lambda(x, m)=\lambda\left(D^{2} u(x, m)\right)=\left(\lambda_{1}, \lambda_{2}, \ldots, \lambda_{n}\right)$ is the eigenvalues of the Hessian matrix $D^{2} u(x, m)$.

Let

$$
\sigma_{l}^{i j}\left(\lambda\left(D^{2} u\right)\right)=\sum_{s=1}^{n} \frac{\partial \sigma_{l}}{\partial \lambda_{s}}\left(\lambda\left(D^{2} u\right)\right) \frac{\partial \lambda_{s}}{\partial r_{i j}}\left(D^{2} u\right) .
$$

If $\lambda(x, m) \in \overline{\Gamma_{l}},(2)$ is degenerate elliptic for $u$ at $(x, m)$, i.e. $\left(\sigma_{l}^{i j}\left(\lambda\left(D^{2} u(x\right.\right.\right.$, $m)))) \geq 0$. And $\sigma_{j}^{\frac{1}{j}}(\lambda(r)), j=1,2, \ldots, l$, is concave for $r$ with $\lambda(r) \in \Gamma_{l}$ (see [5]).

Definition 1.3. A domain $D$ is called uniformly $(l-1)$-convex, if for any $x \in \partial D, \kappa(x)=\left(\kappa_{1}, \ldots, \kappa_{n-1}\right) \in \Gamma_{l-1}$, where $\kappa_{i}, i=1, \ldots, n-1$, denote the principal curvatures of $x \in \partial D$.

From now on we shall always assume 
$\left(H_{3}\right) \quad D$ is uniformly $(l-1)$-convex.

To state our results we require a few suitable notions.

Definition 1.4. A function $u \in C^{0}\left(M_{k}\right)$ is called a viscosity subsolution of (2), if for any $(y, m) \in M_{k}, \xi \in C^{2}\left(M_{k}\right)$ satisfying

$$
u(x, m) \leq \xi(x, m),(x, m) \in M_{k} \quad \text { and } \quad u(y, m)=\xi(y, m),
$$

we have

$$
\sigma_{l}\left(\lambda\left(D^{2} \xi(y, m)\right)\right) \geq f(y, m) .
$$

A function $u \in C^{0}\left(M_{k}\right)$ is called a viscosity supersolution of (2), if for any $(y, m) \in M_{k}$, any $l$-convex function $\xi \in C^{2}\left(M_{k}\right)$ satisfying

$$
u(x, m) \geq \xi(x, m),(x, m) \in M_{k} \quad \text { and } \quad u(y, m)=\xi(y, m),
$$

we have

$$
\sigma_{l}\left(\lambda\left(D^{2} \xi(y, m)\right)\right) \leq f(y, m) .
$$

A function $u \in C^{0}\left(M_{k}\right)$ is called a viscosity solution of (2), if $u$ is both a viscosity subsolution and a viscosity supersolution of (2).

A function $u \in C^{0}\left(M_{k} \cup \partial^{\prime} M_{k}\right)$ is called a viscosity subsolution (supersolution, solution) of (2), (3), if $\mathrm{u}$ is a viscosity subsolution (supersolution, solution) of $(2)$ and satisfies $u(x, m) \leq(\geq,=) \varphi_{m}(x)$ on $\partial^{\prime} M_{k}$ for $m=1,2, \ldots, k$.

In the definition of viscosity subsolution, $\xi$ is not required to be $l$-convex. Urbas proved the definition in which $\xi$ is required to be $l$-convex is equivalent to the definition in which $\xi$ is not required to be $l$-convex, see the Remarks (ii) in [19].

Definition 1.5. A function $u \in C^{0}\left(M_{k}\right)$ is called $l$-convex if in the viscosity sense $\sigma_{j}\left(\lambda\left(D^{2} u(x, m)\right)\right) \geq 0$ in $M_{k}, j=1,2, \ldots, l$.

$u \in C^{0}\left(M_{k}\right)$ is 1-convex if and only if $u$ is $C^{0}$ subharmonic; $u$ is $n$-convex if and only if $u$ is convex.

Similar to the single-valued solutions (see $[17,19]$ ), an $l$-convex function is a viscosity solution of (2) if it is a classical solution of (2). Conversely, an $l$-convex function $u$ is a classical solution of (2) if $u$ is a viscosity solution of (2) and $u$ is of class $C^{2}$.

Our main results in this paper are as follows. Using the Perron method, we obtain an existence theorem.

Theorem 1.6. Suppose $\left(H_{1}\right),\left(H_{2}\right)$ and $\left(H_{3}\right)$ hold, then the Dirichlet problem (2), (3) has at least a bounded l-convex viscosity solution $u \in C^{0}\left(M_{k} \cup \partial^{\prime} M_{k}\right)$.

Theorem 1.7. Let $f \in C^{1}\left(M_{k}\right)$ satisfy $a \leq f \leq b$ for some positive constants $a$ and $b$. If $u \in C^{0}\left(M_{k} \cup \partial^{\prime} M_{k}\right)$ is an l-convex viscosity solution of (2), (3), then $u \in C_{\text {loc }}^{0,1}\left(M_{k}\right)$. $C^{0,1}$.

It would be interesting to see if there is a higher interior regularity than 


\section{Preliminaries}

In this section, we prove some results about the single-valued solutions to Hessian equations which will be used later.

The first one is the weak Minkowsky inequality.

Lemma 2.1. Assume $u \in C^{2}(D), v \in C^{0}(D)$ are l-convex. Then in the viscosity sense

$$
\sigma_{j}^{\frac{1}{j}}\left(\lambda\left(D^{2} u+D^{2} v\right)\right) \geq \sigma_{j}^{\frac{1}{j}}\left(\lambda\left(D^{2} u\right)\right)+\sigma_{j}^{\frac{1}{j}}\left(\lambda\left(D^{2} v\right)\right), \quad \text { in } D
$$

for $j=1,2, \ldots, l$.

Proof. For any $y \in D, \xi \in C^{2}(D)$ satisfying

$$
v(y)=\xi(y), \quad v(x) \leq \xi(x), \quad x \in D,
$$

we have $\lambda\left(D^{2} \xi(y)\right) \in \overline{\Gamma_{l}}$ by virtue of the l-convexity of $v$. Because $\sigma_{j}^{\frac{1}{j}}(\lambda(r)), j=$ $1,2, \ldots, l$ is concave for $r$ when $\lambda(r) \in \overline{\Gamma_{l}}$, then at $y$ we have

$$
\sigma_{j}^{\frac{1}{j}}\left(\lambda\left(\frac{D^{2} u+D^{2} \xi}{2}\right)\right) \geq \frac{1}{2} \sigma_{j}^{\frac{1}{j}}\left(\lambda\left(D^{2} u\right)\right)+\frac{1}{2} \sigma_{j}^{\frac{1}{j}}\left(\lambda\left(D^{2} \xi\right)\right) .
$$

Therefore at $y$,

$$
\sigma_{j}^{\frac{1}{j}}\left(\lambda\left(D^{2} u+D^{2} \xi\right)\right) \geq \sigma_{j}^{\frac{1}{j}}\left(\lambda\left(D^{2} u\right)\right)+\sigma_{j}^{\frac{1}{j}}\left(\lambda\left(D^{2} \xi\right)\right) .
$$

Hence (4) follows.

Next, we always assume that $f \in C^{0}(\bar{D})$ is nonnegative and $\varphi \in C^{0}(\partial D)$.

Lemma 2.2. Suppose $0 \leq f \in C^{0}(\bar{D})$ and $\varphi \in C^{0}(\partial D)$. Then the Dirichlet problem

$$
\begin{array}{rlrl}
\sigma_{l}\left(\lambda\left(D^{2} u\right)\right) & =f(x), \quad & x \in D, \\
u & =\varphi(x), \quad x \in \partial D
\end{array}
$$

has unique l-convex viscosity solution $u \in C^{0}(\bar{D})$.

Proof. Let $\varphi_{i} \in C^{\infty}(\partial D),\left\|\varphi_{i}\right\|_{C^{0}(\partial D)} \leq 2\|\varphi\|_{C^{0}(\partial D)}, \varphi_{i} \rightarrow \varphi$ in $C^{0}(\partial D)$, as $i \rightarrow \infty$, and let $0<f_{i} \in C^{\infty}(\bar{D}),\left\|f_{i}\right\|_{C^{0}(\bar{D})} \leq 2\|f\|_{C^{0}(\bar{D})}, f_{i} \rightarrow f$ in $C^{0}(\bar{D})$, as $i \rightarrow \infty$. Consider the Dirichlet problem

$$
\begin{aligned}
\sigma_{l}\left(\lambda\left(D^{2} u_{i}\right)\right) & =f_{i}(x), \quad x \in D, \\
u_{i} & =\varphi_{i}(x), \quad x \in \partial D .
\end{aligned}
$$

From [5], we know (6), (7) has an $l$-convex solution $u_{i} \in C^{\infty}(\bar{D})$.

We then have, from Lemma 2.1 in [17], for any indices $i, j$, and any $x \in \bar{D}$,

$$
\left|u_{i}(x)-u_{j}(x)\right| \leq C|| f_{i}-f_{j} \|_{C^{0}(\bar{D})}+\max _{\partial D}\left|\varphi_{i}-\varphi_{j}\right| \rightarrow 0, \quad \text { as } i, j \rightarrow \infty .
$$

Consequently, $\left\{u_{i}\right\}$ converges uniformly on $\bar{D}$ to a viscosity solution $u \in C^{0}(\bar{D})$ of (5). The uniqueness can be obtained by the comparison principle. Lemma 2.2 is proved. 
Lemma 2.3. Let $D^{\prime} \subset \subset D$ be an open set. Suppose $V$ is a local bounded function in $D$ and $c$ is a positive constant. Then there exists an l-convex function $\underline{u} \in C^{0}(\bar{D})$ satisfying

$$
\begin{gathered}
\sigma_{l}\left(\lambda\left(D^{2} \underline{u}\right)\right) \geq c, \quad x \in D, \\
\underline{u}=\varphi(x), \quad x \in \partial D, \\
\underline{u} \leq V(x), \quad x \in D^{\prime} .
\end{gathered}
$$

Proof. From Lemma 2.2, we have $l$-convex function $\tilde{\varphi} \in C^{0}(\bar{D})$ satisfying

$$
\begin{gathered}
\sigma_{l}\left(\lambda\left(D^{2} \tilde{\varphi}\right)\right)=1, \quad x \in D, \\
\tilde{\varphi}=\varphi(x), \quad x \in \partial D .
\end{gathered}
$$

Let $\rho \in C^{\infty}(\bar{D})$ (see [5]) be an $l$-convex solution of the Dirichlet problem

$$
\begin{aligned}
\sigma_{l}\left(\lambda\left(D^{2} \rho\right)\right) & =1, \quad x \in D, \\
\rho & =0, \quad x \in \partial D .
\end{aligned}
$$

By the strong maximum principle, $\rho \leq-\rho_{0}$ on $\overline{D^{\prime}}$ for some positive constant $\rho_{0}$. Define

$$
\underline{u}(x)=\tilde{\varphi}(x)+\mu \rho(x), \quad x \in D,
$$

where $\mu$ is a positive constant to be determined. Then $\underline{u}=\varphi$ on $\partial D$ and

$$
\underline{u}=\tilde{\varphi}+\mu \rho \leq \sup _{D^{\prime}} \tilde{\varphi}-\mu \rho_{0} \leq \inf _{D^{\prime}} V \leq V \text {, in } D^{\prime} \text {, if } \mu \text { is large. }
$$

By Lemma 2.1, in the viscosity sense,

$\sigma_{j}\left(\lambda\left(D^{2} \underline{u}\right)\right) \geq \sigma_{j}\left(\lambda\left(D^{2}(\mu \rho)\right)\right)=\mu^{j} \sigma_{j}\left(\lambda\left(D^{2} \rho\right)\right) \geq 0, \quad x \in D, \quad j=1,2, \ldots, l$.

Hence $\underline{u} \in C^{0}(\bar{D})$ is l-convex and by choosing $\mu$ large enough,

$$
\sigma_{l}\left(\lambda\left(D^{2} \underline{u}\right)\right) \geq \mu^{l} \geq c, \quad x \in D .
$$

The proof of Lemma 2.3 is completed.

Lemma 2.4. Let $\Omega \subset \subset D$ be an open set. Assume l-convex functions $u \in$ $C^{0}(D), v \in C^{0}(\bar{\Omega})$ satisfy, respectively

$$
\begin{array}{ll}
\sigma_{l}\left(\lambda\left(D^{2} u\right)\right) \geq f(x), & x \in D, \\
\sigma_{l}\left(\lambda\left(D^{2} v\right)\right) \geq f(x), & x \in \Omega .
\end{array}
$$

and

$$
u=v, x \in \partial \Omega, \quad u \leq v, \quad x \in \bar{\Omega} .
$$

Set

$$
w(x)=\left\{\begin{array}{l}
u(x), x \in D \backslash \Omega, \\
v(x), x \in \Omega .
\end{array}\right.
$$

Then $w \in C^{0}(D)$ is an l-convex function in $D$ and satisfies in the viscosity sense

$$
\sigma_{l}\left(\lambda\left(D^{2} w\right)\right) \geq f(x), \quad x \in D
$$


Proof. Let $y \in D, \xi \in C^{2}(D)$ satisfying

$$
w(y)=\xi(y), \quad w(x) \leq \xi(x), \quad x \in D .
$$

If $y \in D \backslash \Omega$, we have $u(y)=\xi(y)$, and $u \leq \xi$ in $D$ by (8), (9). Therefore

$$
\sigma_{l}\left(\lambda\left(D^{2} \xi(y)\right)\right) \geq f(y) .
$$

If $y \in \Omega$, we have $v(y)=\xi(y)$, and $v \leq \xi$ in $\Omega$. Therefore

$$
\sigma_{l}\left(\lambda\left(D^{2} \xi(y)\right)\right) \geq f(y) .
$$

This completes the proof of Lemma 2.4.

\section{Existence and regularity of bounded solutions}

In this section, we prove Theorem 1.6. We first introduce a comparison principle in $M_{k}$ (see [4]).

Lemma 3.1. Let $u, v \in C^{0}\left(M_{k}\right) \cap L^{\infty}\left(M_{k}\right)$ satisfy in the viscosity sense $\Delta u \geq$ $0 \geq \Delta v$ in $M_{k}$ and

$$
\liminf _{\operatorname{dist}\left((x, m), \partial^{\prime} M_{k}\right) \rightarrow 0}(u(x, m)-v(x, m)) \leq 0,
$$

then $u \leq v$ in $M_{k}$.

Proof of Theorem 1.6. We divide the proof into three steps.

Step 1 We construct a viscosity subsolution of (2).

Let $d=\operatorname{diam} D$, and $h \in C^{0}\left(M_{k}\right) \cap L^{\infty}\left(M_{k}\right)$ (see [4]) satisfy

$$
\begin{aligned}
\Delta h & =0, \quad(x, m) \in M_{k}, \\
h & =\varphi_{m}(x), \quad(x, m) \in \partial^{\prime} M_{k} .
\end{aligned}
$$

Fix $x_{0} \in D$, let $P(x)=A\left|x-x_{0}\right|^{2}-B$, where $A, B$ are constants to be determined. Choose $A=A(n, l, b)$ and then $B=B\left(n, l, b, d, \inf _{M_{k}} h\right)$ sufficiently large such that

$$
\begin{gathered}
\sigma_{l}\left(\lambda\left(D^{2} P\right)\right)=\sum_{i_{1}<\cdots<i_{l}}(2 A)^{l}=C_{n}^{l}(2 A)^{l} \geq b, \quad x \in D, \\
P(x) \leq A d^{2}-B<\inf _{M_{k}} h, \quad X \in \bar{D}
\end{gathered}
$$

where $C_{n}^{l}=n ! /(l !(n-l) !)$.

From Lemma 2.3 , for $m=1,2, \ldots, k$, there exist $l$-convex functions $\underline{u}_{m} \in$ $C^{0}(\bar{D})$ satisfying

$$
\begin{aligned}
\sigma_{l}\left(\lambda\left(D^{2} \underline{u}_{m}\right)\right) & \geq b, \quad x \in D, \\
\underline{u}_{m} & =\varphi_{m}(x), \quad x \in \partial D, \\
\underline{u}_{m} & \leq P(x), \quad x \in D^{\prime},
\end{aligned}
$$

where $D^{\prime}$ is an open set satisfying $\Sigma \subset \subset D^{\prime} \subset \subset D$.

Define

$$
\underline{u}(x, m)=\max \left\{\underline{u}_{m}(x), P(x)\right\}, \quad x \in D .
$$


Then

$$
\underline{u}(x, m)=P(x), \quad x \in D^{\prime},
$$

and from [13], $\underline{u} \in C^{0}\left(M_{k} \cup \partial^{\prime} M_{k}\right)$ is an $l$-convex viscosity subsolution of (2). By $(12), P \leq h=\varphi_{m}=\underline{u}_{m}$ on $\partial D$ and then $\underline{u}(x, m)=\varphi_{m}(x)$ on $\partial D$.

Step 2 We define the Perron solution of (2)

Let $\mathbb{S}$ denote the set of $l$-convex viscosity subsolutions $v \in C^{0}\left(M_{k} \cup \partial^{\prime} M_{k}\right)$ of (2), (3) which satisfy

$$
\limsup _{x \rightarrow \bar{x}} \max _{1 \leq m \leq k}(v(x, m)-h(x, m)) \leq 0, \quad \bar{x} \in \Gamma .
$$

Clearly $\underline{u} \in \mathbb{S}$, and then $\mathbb{S} \neq \emptyset$. Define

$$
u(x, m)=\sup \{v(x, m) \mid v \in \mathbb{S}\}, \quad(x, m) \in M_{k} .
$$

Then from [12], $u \in C^{0}\left(M_{k} \cup \partial^{\prime} M_{k}\right)$ and from [13], $u$ is an $l$-convex viscosity subsolution of (2). Because $\underline{u} \leq u$ in $M_{k}$ and $\underline{u}=\varphi_{m}$ on $\partial D$ for $m=1,2, \ldots, k$, then

$$
u(x, m)=\varphi_{m}(x), \quad(x, m) \in \partial^{\prime} M_{k} .
$$

Step 3 We prove $u$ is a viscosity supersolution of (2)

For any $(y, m) \in M_{k}, l$-convex function $\xi \in C^{2}\left(M_{k}\right)$ satisfying

$$
u(y, m)=\xi(y, m), \quad \text { and } \quad u(x, m) \geq \xi(x, m), \quad(x, m) \in M_{k},
$$

choose a ball $B=B_{r}(y)$ such that $\bar{B} \subset D \backslash \Gamma$. The lifting of $B$ into $M_{k}$ is the union of $k$ disjoint balls denoted as $\left\{B^{(i)}\right\}_{i=1}^{k}$. In each ball $B^{(i)}$, by Lemma 2.2,

$$
\begin{aligned}
\sigma_{l}\left(\lambda\left(D^{2} \tilde{u}\right)\right) & =f(x, m), & & (x, m) \in B^{(i)}, \\
\tilde{u} & =u(x, m), & & (x, m) \in \partial B^{(i)}
\end{aligned}
$$

has an $l$-convex viscosity solution $\tilde{u} \in C^{0}\left(\overline{B^{(i)}}\right)$. From the comparison principle,

$$
u \leq \tilde{u},(x, m) \in B^{(i)} .
$$

Define in $M_{k}$,

$$
w(x, m)=\left\{\begin{array}{l}
\tilde{u}(x, m),(x, m) \in B^{(i)} \\
u(x, m),(x, m) \in M_{k} \backslash\left\{B^{(i)}\right\}_{i=1}^{k}
\end{array}\right.
$$

Because

$$
w(x, m)=u(x, m)=\varphi_{m}(x), \quad x \in \partial D,
$$

then by Lemma 2.4 and (16), we know $w$ is an $l$-convex viscosity subsolution of $(2),(3)$.

If $w$ satisfies $(14)$, then $w \in \mathbb{S}$. In fact, in the viscosity sense

$$
\Delta w \geq 0=\Delta h, \text { in } M_{k},
$$

and

$$
w=\varphi_{m}=h \text { on } \partial^{\prime} M_{k}
$$


By Lemma 3.1,

$$
w \leq h \quad \text { in } M_{k}
$$

and then $w$ satisfies (14).

By the definition of $u, u \geq w$ in $M_{k}$, and then $\tilde{u} \leq u$ in $B^{(i)}$. Considering (16), we obtain

$$
\tilde{u}=u,(x, m) \in B^{(i)},
$$

and hence

$$
\tilde{u}(y, m)=\xi(y, m), \quad \text { and } \quad \tilde{u}(x, m) \geq \xi(x, m), \quad(x, m) \in B^{(i)} .
$$

But $\tilde{u}$ satisfies (15), we have

$$
\sigma_{l}\left(\lambda\left(D^{2} \xi(y, m)\right)\right) \leq f(y, m) .
$$

It follows that in the viscosity sense $u$ satisfies

$$
\sigma_{l}\left(\lambda\left(D^{2} u\right)\right) \leq f, \quad \text { in } M_{k} .
$$

This completes the proof of Theorem 1.6.

In the following, we prove the interior Lipschitz regularity of the multivalued solutions.

Proof of Theorem 1.7. For any $x_{0} \in D \backslash \Gamma$, fix $\varepsilon>0$ such that $\bar{B}=\bar{B}_{\varepsilon}\left(x_{0}\right) \subset$ $D \backslash \Gamma$. The lifting of $B$ into $M_{k}$ is the union of $k$ disjoint balls denoted as $\left\{B^{(i)}\right\}_{i=1}^{k}$. In each ball $B^{(i)}$, by Lemma 2.2 ,

$$
\begin{aligned}
\sigma_{l}\left(\lambda\left(D^{2} u^{(i)}\right)\right) & =f(x, m), & & (x, m) \in B^{(i)}, \\
u^{(i)} & =u(x, m), & & (x, m) \in \partial B^{(i)}
\end{aligned}
$$

has an $l$-convex viscosity solution $u^{(i)} \in C^{0}\left(\overline{B^{(i)}}\right)$, where $|i-m| \leq 1$ (the $k$ th sheet can be looked as the 0th sheet). From the comparison principle,

$$
u=u^{(i)},(x, m) \in B^{(i)} .
$$

We know, by Trudinger and Wang [18] or Urbas [19], $u^{(i)} \in C^{0,1}\left(B^{(i)}\right)$. And therefore, from (19), we have $u \in C_{l o c}^{0,1}\left(M_{k}\right)$.

\section{Acknowledgments}

The authors would like to thank Professor Luis Caffarelli for his suggestions, and Professor Yanyan Li for several very informative conversations in the development of this work. This work is supported by National Natural Science Foundation of China (10671022) and Doctoral Programme Foundation of Institute of Higher Education (20060027023). 


\section{References}

[1] Almgren, F.: F.Almgren's big regularity paper. Q-valued functions minimizing Dirichlet's integral and the regularity of area-minimizing rectifiable currents up to codimension 2. World Scientific Monograph Series in Mathematics, 1. World Scientific Publishing Co., Inc., River Edge, NJ, 2000

[2] Caffarelli, L.: Certain multiple valued harmonic functions. Proc. Am. Math. Soc. 54, 90-92 (1976)

[3] Caffarelli, L.: On the Hölder continuity of multiple valued harmonic functions. Indiana Univ. Math. J. 25, 79-84 (1976)

[4] Caffarelli, L., Li, Y.Y.: Some multi-valued solutions to Monge-Ampère equations. Commun. Anal. Geom. 14, 411-441 (2006)

[5] Caffarelli, L., Nirenberg, L., Spruck, J.: The Dirichlet problem for nonlinear second-order elliptic equations. III. Functions of the eigenvalues of the Hessian. Acta Math. 155, 261-301 (1985)

[6] Evans, G.C.: A necessary and sufficient condition of Wiener. Am. Math. Month. 54, 151-155 (1947)

[7] Evans, G.C.: Surfaces of minimal capacity. Proc. Natl. Acad. Sci. USA 26, 489$491(1940)$

[8] Evans, G.C.: Lectures on multiple valued harmonic functions in space. Univ. Calif. Publ. Math. (N.S.) 1, 281-340 (1951)

[9] Ferrer, L., Martínez, A., Milán, F.: An extension of a theorem by K. Jörgens and a maximum principle at infinity for parabolic affine spheres. Math. Z. 230, 471486 (1999)

[10] Gosse, L., Jin, S., Li, X.: Two moment systems for computing multiphase semiclassical limits of the Schrödinger equation. Math. Models Methods Appl. Sci. 13, 1689-1723 (2003)

[11] Izumiya, S., Kossioris, G.T., Makrakis, G.N.: Multivalued solutions to the eikonal equation in stratified media. Q. Appl. Math. 59, 365-390 (2001)

[12] Ishii, H.: Perron's method for Hamilton-Jacobi equations. Duke Math. J. 55, 369$384(1987)$

[13] Ishii, H., Lions, P.L.: Viscosity solutions of fully nonlinear second-order elliptic partial differential equations. J. Differ. Equ. 83, 26-78 (1990)

[14] Jin, S., Osher, S.: A level set method for the computation of multivalued solutions to quasi-linear hyperbolic PDEs and Hamilton-Jacobi equations. Commun. Math. Sci. 1, 575-591 (2003)

[15] Jin, S., Liu, H., Osher, S., Tsai, Y.: Computing multivalued physical observables for the semiclassical limit of the Schrödinger equation. J. Comput. Phys. 205, 222-241 (2005) 
[16] Levi, G.: Generalization of a spatial angle theorem. Translated from the English by Ju. V. Egorov. Uspehi Mat. Nauk 26(2):158, 199-204 (1971) (Russian)

[17] Trudinger, N.S.: The Dirichlet problem for the prescribed curvature equations. Arch. Ration. Mech. Anal. 111, 153-179 (1990)

[18] Trudinger, N.S., Wang, X.J.: Hessian measures. II. Ann. Math. 150, 579604 (1999)

[19] Urbas, J.I.E.: On the existence of nonclassical solutions for two class of fully nonlinear elliptic equations. Indiana Univ. Math. J. 39, 355-382 (1990)

Limei Dai

School of Mathematics and Information Science,

Weifang University,

Shandong 261061,

People's Republic of China

Jiguang Bao $(\varangle)$

School of Mathematical Sciences,

Beijing Normal University,

Laboratory of Mathematics and Complex Systems,

Ministry of Education,

Beijing 100875,

People's Republic of China

e-mail: jgbao@bnu.edu.cn

Received: 17 October 2010.

Accepted: 19 January 2011. 